\title{
An ASM specification of C\# threads and the .NET memory model
}

\author{
Robert F. Stärk ${ }^{1}$ and Egon Börger ${ }^{2}$ \\ 1 Computer Science Department, ETH Zürich \\ staerk@inf .ethz.ch \\ 2 Dipartimento di Informatica, Università di Pisa \\ boerger@di.unipi.it
}

\begin{abstract}
We present a high-level ASM model of $\mathrm{C} \#$ threads and the .NET memory model. We focus on purely managed, fully portable threading features of $\mathrm{C \#}$. The sequential model interleaves the computation steps of the currently running threads and is suitable for uniprocessors. The parallel model addresses problems of true concurrency on multiprocessor systems. The models provide a sound basis for the development of multi-threaded applications in C\#. The thread and memory models complete the abstract operational semantics of $\mathrm{CH}$ in [2].
\end{abstract}

\section{Introduction}

Modern object-oriented programming languages like Java or C\# support multithreaded programming. They allow several threads to run concurrently sharing objects on the heap in the same address space. Each thread has its own frame stack, program counter, local variables and registers. The languages have special syntactical constructs for synchronization. Java has a synchronized statement and synchronized methods, while $\mathrm{C} \#$ has a lock statement and several attributes that can be applied to classes and methods to control their run-time synchronization behavior.

Although the C\# programming languages supports multi-threaded programming directly via special syntax, the underlying thread model is poorly documented and still considered to be part of the library. The Ecma standards for C\# [4] and the Common Language Infrastructure [5] contain only a few paragraphs about threads. For example, the lock statement is defined in [4, §15.22] by a reduction to the library functions Monitor. Enter and Monitor. Exit which are not further specified there. Important issues, such as the order of writes to volatile and non-volatile fields, are just briefly mentioned in two paragraphs in $[4, \S 10.10, \S 17.4 .3]$. Hence, a program developer has to rely solely on the class library documentation that comes with Microsoft's .NET framework Software Development Kit [11]. Unfortunately, that documentation is not very precise with respect to threads, locks and memory issues. Moreover, it is not identical with the (XML) specification of the types that comprise the standard libraries in [5, Partition IV, Profiles and Libraries]. For example, specifications 
of Thread.Interrupt, Thread.Suspend and Thread.Resume are not included in $[5]$.

If a programmer cannot rely on a simple and precise thread model, the task of writing reliable multi-threaded applications that are correctly synchronized and free of data races and deadlocks becomes very difficult and tedious. Multithreaded programs depend on the scheduling policy of underlying run-time system and therefore synchronization errors are difficult to reproduce and to debug. Moreover, certain problems may only occur under heavy threading stress in production environments like web services which cannot be simulated during the development cycle. Tools that statically analyze multi-threaded programs for synchronization problems are in general neither sound nor complete. Nevertheless, in some cases the may report a high percentage of all possible conflicts (see [19]).

The Java Language Specification [7, Ch. 17] devotes a whole chapter to threads and locks. However, that specification has been found to be hard to understand and has subtle, often unintended, implications. Therefore, the Java community has proposed a new specification of the semantics of threads and locks often referred to as the New Java Memory Model [10]. Whether the new specification is easier to understand may be doubted. It justifies at least most of the common compiler optimizations which were prohibited by the old one. For a comparison and analysis of the different proposals we refer to [1].

The specification of threads in this article extends the modular definition of the semantics of $\mathrm{C} \#$ in [2] by a new module $\mathrm{C} \#_{\mathcal{T}}$ for multi-threaded $\mathrm{C \#}$. We focus on purely managed, fully portable threading features of $\mathrm{C \#}$ and the .NET common language runtime. We do not consider the .NET equivalents of Win32 threading primitives such as WaitHandle and their derived classes. We also do not model asynchronous delegates and synchronization domains. The starting point of our model has been the thread model for Java in [17]. That model however is only correct for uniprocessor systems and does not address problems of true concurrency.

For basic terminology on Abstract State Machines we refer the reader to [3,8].

\section{Threads in Microsoft's .NET framework}

The thread related features of $\mathrm{C \#}$ are collected in the System. Threading namespace (see Fig. 1). The namespace contains the delegate type ThreadStart that denotes the type of functions with zero arguments and return type void. The most important classes of the namespace are the Thread and Monitor classes. Several thread related exception classes derived from SystemException are also declared in the namespace.

A thread can be in one or more states of the ThreadState enumeration (listed in Fig. 1). Unfortunately, the documentation does not state clearly which combinations of states are allowed for a thread and which are not. Moreover, some of the states are not real execution states of a thread but just boolean flags. The Background state, for example, tells the run-time system that it can kill the 
thread and exit when all non-background threads have terminated (similar to the Daemon property of threads in Java). Other states, like StopRequested, are for internal use only and should not be exposed to the programmer in a public enumeration. The Aborted state has a rather obscure meaning (see below). If there is an AbortRequested, why is there no InterruptRequested?

The ThreadState property of the Thread class returns a snapshot containing the states of a thread as a bitset. This information, however, cannot be used for synchronization purposes, since it may already been outdated when it is obtained. Therefore, we do not model the ThreadState property below and use a different set of execution states in our model.

Threads are represented in C\# by instances of class Thread in Fig. 2. Unlike in Java, this class is sealed (final in Java terminology) and cannot be subclassed. The constructor of the class takes a pointer to a ThreadStart function which will be executed when the new thread is started. The two static methods of the class, Sleep and ResetAbort, are implicitly called on the current thread.

The constructor of class Monitor in Fig. 2 is private, which means that no instances of this class can be created. The reason is, that in C\# (like in Java) every object reference can be used as a monitor and therefore there is no need to create special monitors. The Monitor class contains only static methods. Its Wait, Pulse and PulseAll methods are similar to Java's wait, notify and notifyAll methods of class java.lang. Object.

The Enter and Exit methods of the Monitor class are used to syntactically reduce the lock statement of $\mathrm{C} \#$ (where $o$ is a fresh local variable):

$$
\text { lock (exp)stm } \Longrightarrow\left\{\begin{array}{l}
\text { object } o=\exp ; \\
\text { Monitor.Enter }(o) ; \\
\text { try }\{\operatorname{stm}\} \\
\text { finally }\{\text { Monitor.Exit }(o) ;\}
\end{array}\right.
$$

Unlike in Java, the Monitor.Enter and Monitor.Exit methods can be called explicitly in $\mathrm{C} \#$ programs and hence $\mathrm{C} \#$ cannot guarantee that a thread holds no more locks when it has terminated.

\section{An ASM model for threads on uniprocessors}

Whenever in $\mathrm{C} \#$ an object is created on the heap, it gets two additional overhead fields associated with it. The first field is a pointer to the object's method table. This pointer makes it possible to obtain the run-time type (exact type) of the object. The second field contains an index of a SyncBlock. SyncBlocks are associated with an object on the fly when the object is used as a monitor. A SyncBlock structure contains information that is used for thread synchronization (cf. [14]). 
When an object ref is used as a Monitor the functions are initialized as follows:

$$
\begin{array}{ll}
\text { lockOwner }(\text { ref }):=\text { None } & \text { readyQueue }(\text { ref }):=[] \\
\text { lockCount }(\text { ref, thread) }:=\text { Undef } & \text { waitQueue }(\text { ref }):=[]
\end{array}
$$

The possible execution states of a thread (explained in detail below) are:

$$
\begin{gathered}
\text { ExecState }::=\text { Unstarted } \mid \text { Active } \mid \text { Suspended } \mid \text { Sleeping } \mid \text { Joined } \\
\mid \text { Syncing } \mid \text { Waiting } \mid \text { Pulsed } \mid \text { Dead }
\end{gathered}
$$

The function execState returns the unique execution state of a thread.

$$
\text { execState }: \text { Thread } \rightarrow \text { ExecState }
$$

Every thread has several attributes. The joinSet comprises the threads that are joined to the current thread and are waiting for its termination. The wakeup Time stores the time when the current thread expires. The monObj is the monitor the current thread is waiting for or wants to acquire. The joinedThread is another thread on which the current thread is joined. Moreover, several flags indicate whether an abort has been requested or initiated, whether an interrupt has been requested, or whether a suspend has been requested.

$$
\begin{array}{ll}
\text { joinSet: Thread } \rightarrow \text { Powerset }(\text { Thread }) & \text { abortRequested: Thread } \rightarrow \text { Bool } \\
\text { wakeup Time: Thread } \rightarrow \mathbb{N} \cup\{\infty\} & \text { abortInitiated: Thread } \rightarrow \text { Bool } \\
\text { monObj: Thread } \rightarrow \text { Monitor } & \text { interruptRequested: Thread } \rightarrow \text { Bool } \\
\text { joinedThread: Thread } \rightarrow \text { Thread } & \text { suspendRequested: Thread } \rightarrow \text { Bool }
\end{array}
$$

When an object ref of type Thread is created, the dynamic functions are initialized as follows:

$$
\begin{array}{ll}
\text { joinSet }(\text { ref }):=\emptyset & \text { abortRequested }(\text { ref }):=\text { False } \\
\text { wakeupTime }(\text { ref }):=\text { Undef } & \text { abortInitiated }(\text { ref }):=\text { False } \\
\text { monObj }(\text { ref }):=\text { Undef } & \text { interruptRequested }(\text { ref }):=\text { False } \\
\text { joinedThread }(\text { ref }):=\text { Undef } & \text { suspendRequested }(\text { ref }):=\text { False } \\
\text { execState }(\text { ref }):=\text { Unstarted } &
\end{array}
$$

The local state of a thread comprises a frame stack of activation records, the currently executed method, the current position in the method body (program counter), the local environment and the already computed values of expressions (operand stack).

$$
\begin{array}{ll}
\text { frames: } \text { Thread } \rightarrow \text { List }(\text { Frame }) & \text { locals: Thread } \rightarrow(\text { Loc } \rightarrow \text { Adr }) \\
\text { meth }: \text { Thread } \rightarrow \text { Meth } & \text { values }: \text { Thread } \rightarrow(\text { Pos } \rightarrow \text { Result }) \\
\text { pos: Thread } \rightarrow \text { Pos } &
\end{array}
$$

The current thread is denoted by 'self' in the ASM rules below.

Fig. 3 shows a classification of the execution states of a thread and relates them to the items of the ThreadState enumeration in Fig. 1. A thread is Running if it is not Unstarted and not already Dead. A thread is considered to be Passive (or WaitSleepJoin) if it is Running but neither Active nor Suspended. 


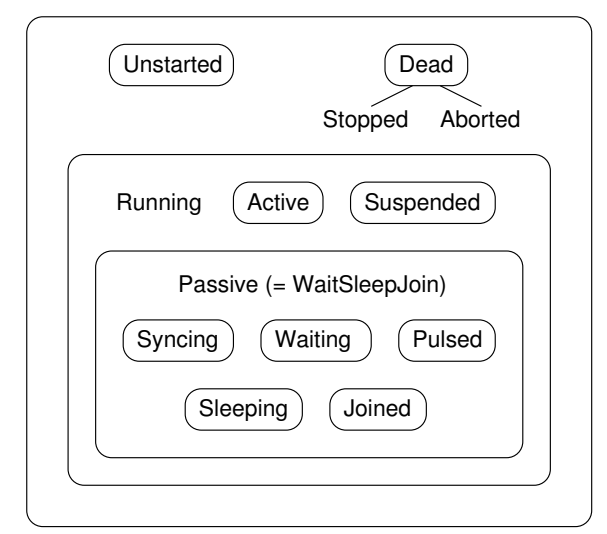

Fig. 3. The execution states of a thread.

$$
\begin{aligned}
& \text { Running }(\text { thread }) \Longleftrightarrow \text { execState }(\text { thread }) \notin\{\text { Unstarted,Dead }\} \\
& \text { Passive }(\text { thread }) \Longleftrightarrow \text { WaitSleepJoin }(\text { thread }) \Longleftrightarrow \\
& \text { execState }(\text { thread }) \in\{\text { Syncing, Waiting, Pulsed, Sleeping, Joined }\}
\end{aligned}
$$

The items Stopped and Aborted of the ThreadState enumeration can be obtained as follows:

$$
\begin{aligned}
& \text { Stopped }(\text { thread }) \Longleftrightarrow \text { execState }(\text { thread })=\text { Dead } \wedge \neg \text { abortRequested }(\text { thread }) \\
& \text { Aborted }(\text { thread }) \Longleftrightarrow \text { execState }(\text { thread })=\text { Dead } \wedge \text { abortRequested }(\text { thread })
\end{aligned}
$$

The reason for this separation is not known to us.

\subsection{An overview of the model}

Fig. 4 and 5 contain diagrams for the execution states of a thread. Methods that are invoked by another thread on the current thread are displayed in grey boxes, whereas methods invoked by the current thread itself are put into white boxes. If there is no outgoing arrow for a thread method from an execution state, then this can mean either that such an invocation is not possible, e.g. since a static method can only be invoked by an active thread, or that the invocation is not allowed and throws a ThreadStateException.

The main rule of the ASM model for uniprocessors in Sect. 3.5 below is the rule ExecSequentialCsharp. It uses the rule ExecCsharp of the ASM model in [2] which executes one computation step of a single-threaded C\# program. The rule ExECCsharp has to be parameterized by the current thread in order to extend the model of [2] to multiple threads. The argument thread of ExecCsharp becomes then the value of 'self' in the rules of [2]. The component EXECCSHARP $T$ of EXecCsharP which has been left open in [2] has to be defined as follows:

$$
\operatorname{ExeCCsharp}_{T} \equiv \text { ExecCsharpStm }_{T}
$$




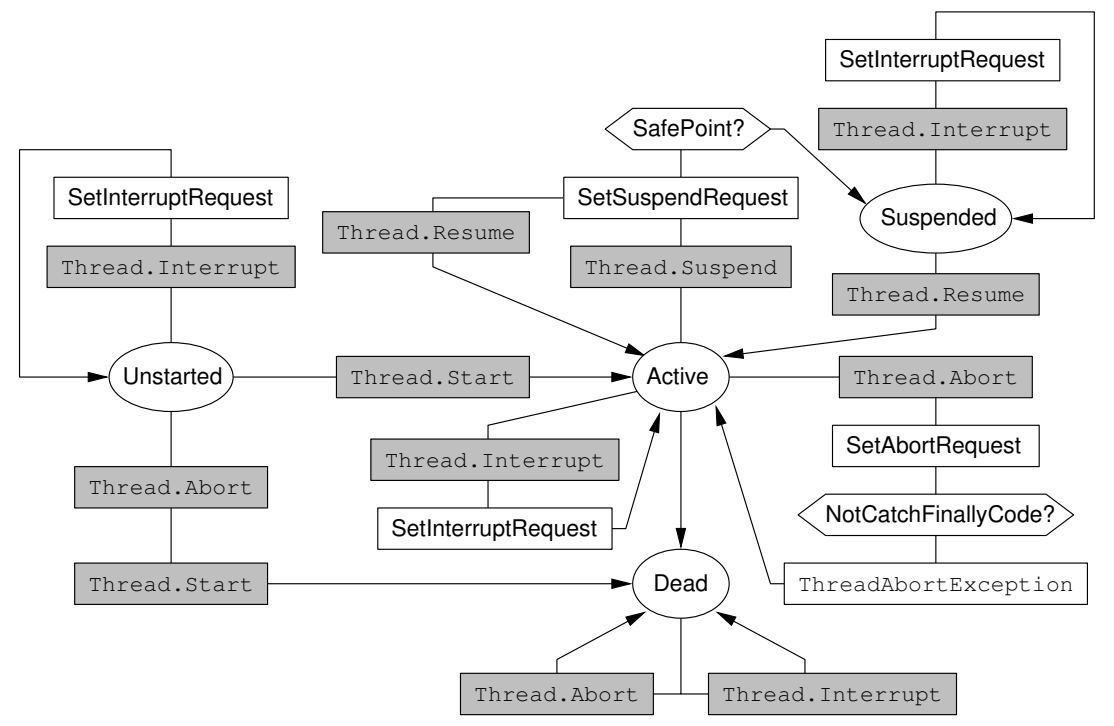

Fig. 4. Methods invoked by other threads on the current thread.

\subsection{The methods of the Thread class}

When a thread is created by invoking the constructor of the Thread class, its execution state is Unstarted. The new thread is later started by invoking the Thread.Start method. A thread can only be started once, otherwise a ThreadStateException exception is thrown. If there has already been an abort requested for the thread, its execution state is immediately changed to Dead. Otherwise, the execution state of the thread is updated to Active and its local state is initiated. The new thread now runs concurrently with the thread that invoked the Thread. Start method. The Y $\operatorname{IELDUP}($ Norm $)$ means that the Thread. Start method returns without blocking.

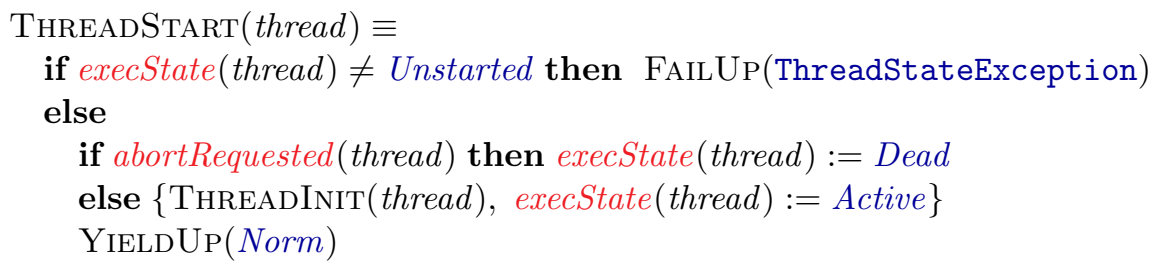

When a thread is created, a delegate of type ThreadStart has to be provided to the constructor of the Thread class. This delegate is later invoked, when the thread is started. Technically, this means the new thread executes the Invoke method of the ThreadStart delegate. If the invocation list of that delegate consists of a single method, then this method is executed. Otherwise, the methods of the invocation list are executed sequentially. 


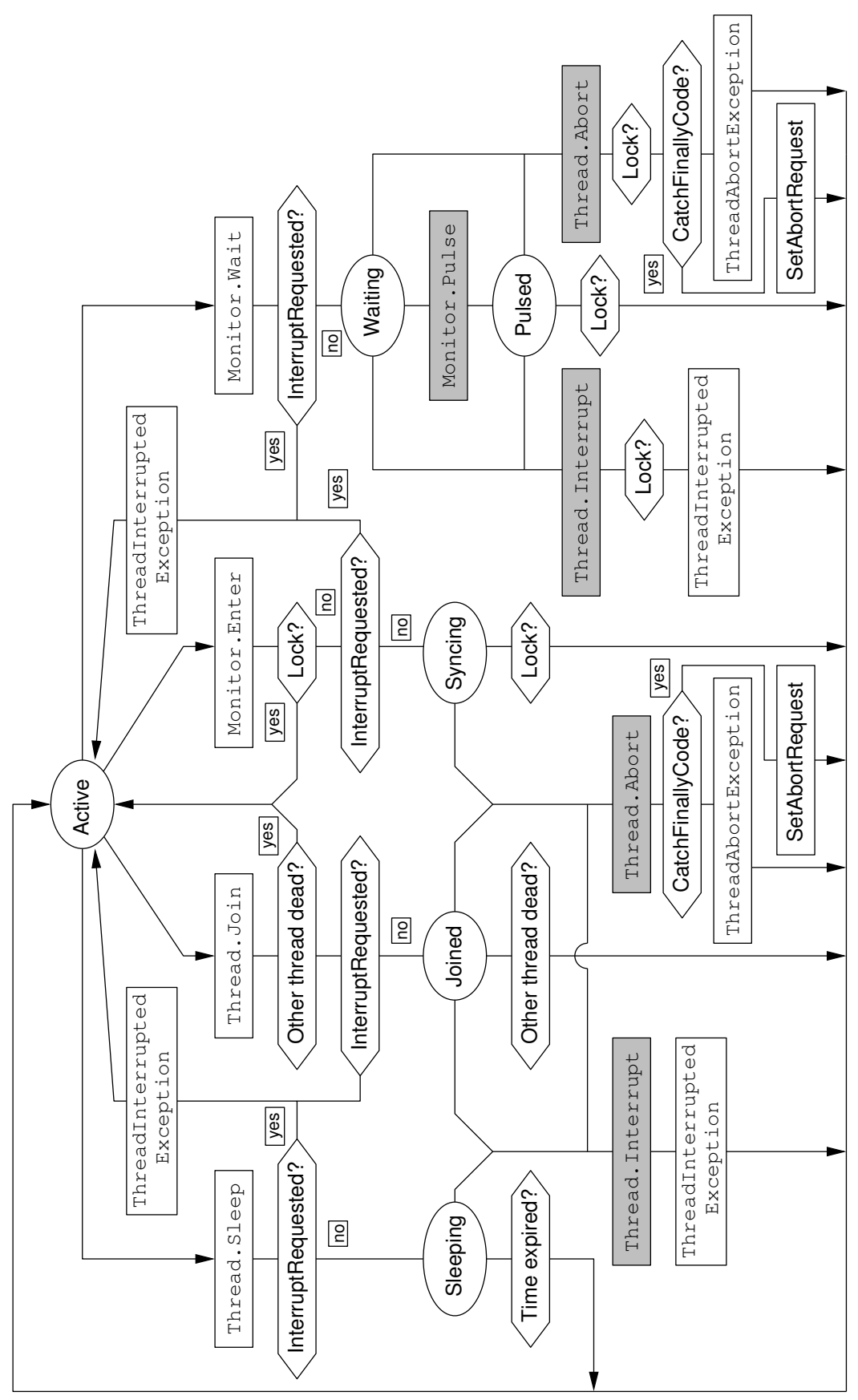

Fig. 5. Methods invoked by the current thread. 


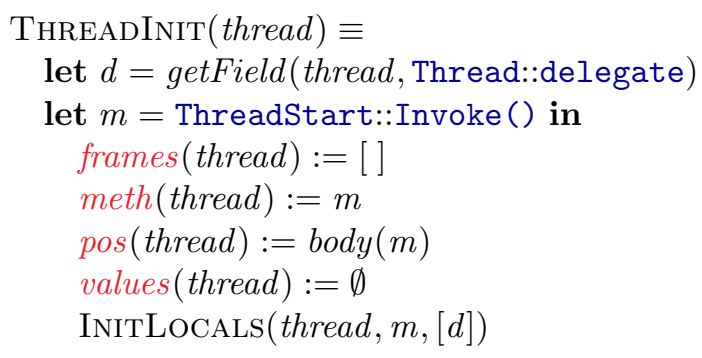

The InitiLocals macro initializes the local environment of the method, for example it assigns the delegate $d$ to the this parameter of Invoke.

The Thread. Join method puts the current thread into the join set of another thread and changes the execution state of the current thread from Active to Joined. Like every thread method that takes a timeout argument it checks first whether the argument is in the correct range. If an interrupt has been requested, then Thread.Join throws a ThreadInterruptedException instead of joining (Mono 0.26 ignores the interrupt request [12]).

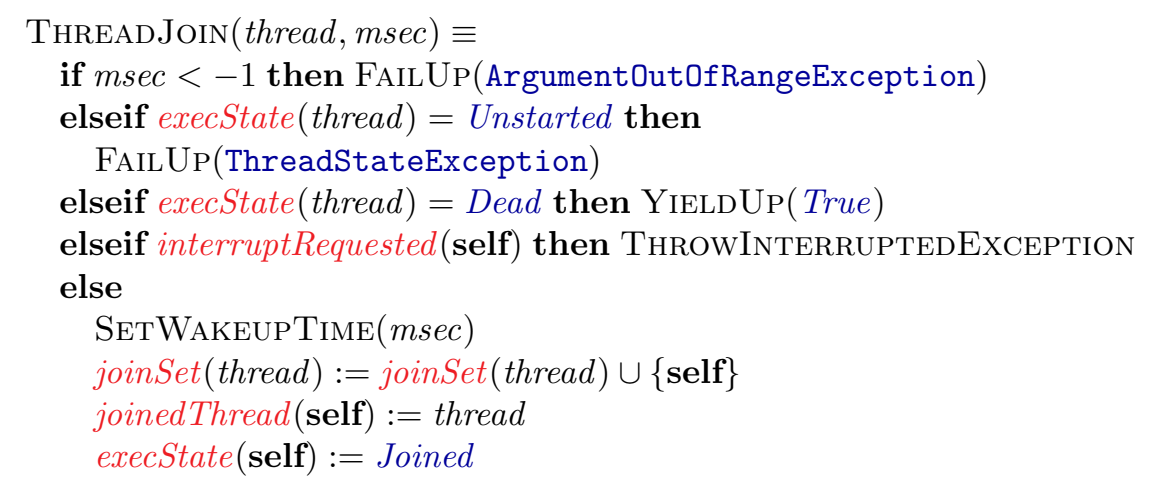

The thread will become active again, when the other thread has terminated or msec milliseconds have passed. An argument of -1 milliseconds means an infinite amount of time.

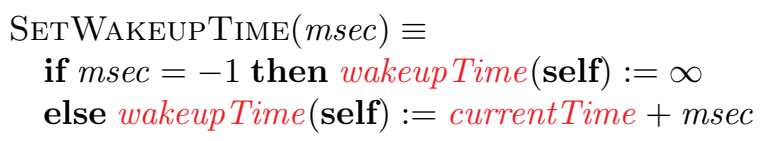

When an ThreadInterruptedException is thrown, the interrupt request of the current thread is cleared.

\section{THROWINTERRUPTEDEXCEPTION $\equiv$ FAILUP(ThreadInterruptedException) interruptRequested(self) $:=$ False}

When the Thread.Join method returns, it indicates with a boolean result, whether the other thread is dead. If the other thread is not dead, then it follows from the definition of the predicate Expired and the WAKEUP rule in Sect. 3.5 that the amount of time has expired. 


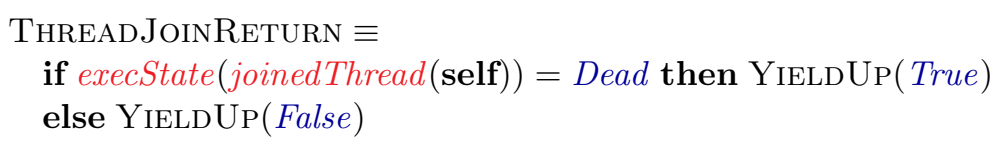

The Thread.Sleep method puts the current thread to sleep for the specified amount of milliseconds. The execution state of the current thread is changed from Active to Sleeping. If an interrupt has been requested, the current thread throws a ThreadInterruptedException instead of going to sleep.

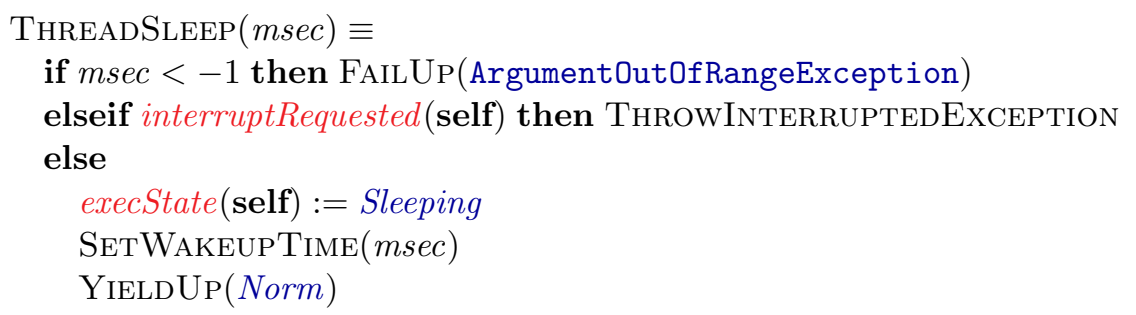

In order to abort another thread with the Thread.Abort method, the current thread needs the appropriate security permission. If the other thread is suspended, a ThreadStateException is thrown, although the documentation [11] says that in that case the other thread is resumed by the system. Otherwise Thread.Abort sets an abort request for the other thread. The effect of this request is that a ThreadAbortException is thrown asynchronously in the other thread (see Sect. 3.5).

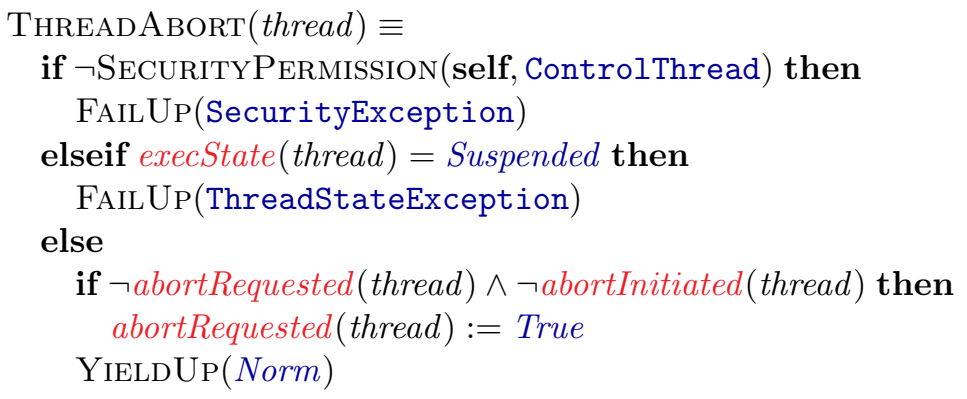

The static method Thread.ResetAbort can be invoked by the current thread to cancel the automatic re-throwing of a ThreadAbortException at the end of catch blocks. It clears the flag that indicates that the abort has been initiated. Only threads that have the appropriate security permission can cancel an abort. The documentation [11] says that the method throws a ThreadStateException if the method was not invoked on the current thread. This can never happen, since it is a static method.

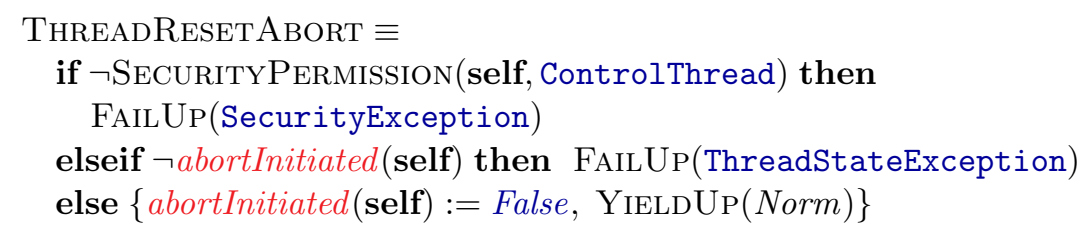


The Thread.Interrupt method sets an interrupt request for another thread. The effect of the request is that a ThreadInterruptedException is injected into the other thread, if it is in a passive state (see Sect. 3.5). Otherwise, the exception is thrown by the other thread, when it changes its execution state from running into a passive state. If the other thread stays active forever, the interrupt request is ignored.

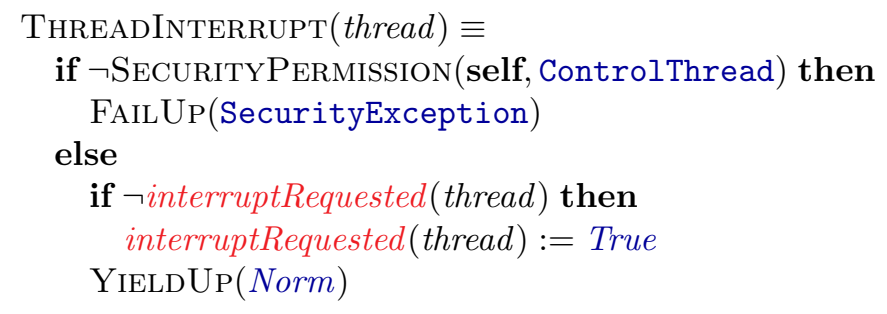

The Thread.Suspend method sets a suspend request for another thread, if it is running. The request will asynchronously be processed by the run-time system (see Sect. 3.5).

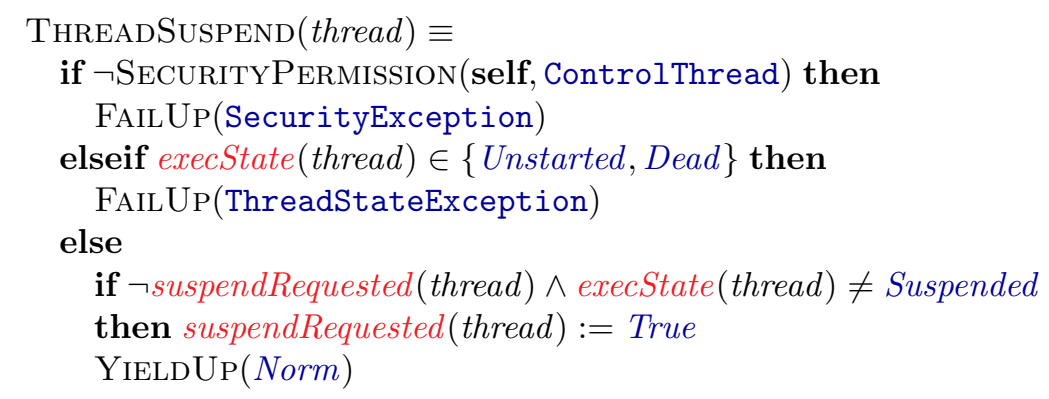

A thread can be resumed by invoking Thread.Resume only if it is suspended or a suspend request is pending. If the thread is suspended, then its execution state is changed back to active such that it can be scheduled for execution by the run-time system again. If a suspend has been requested, the request is cleared.

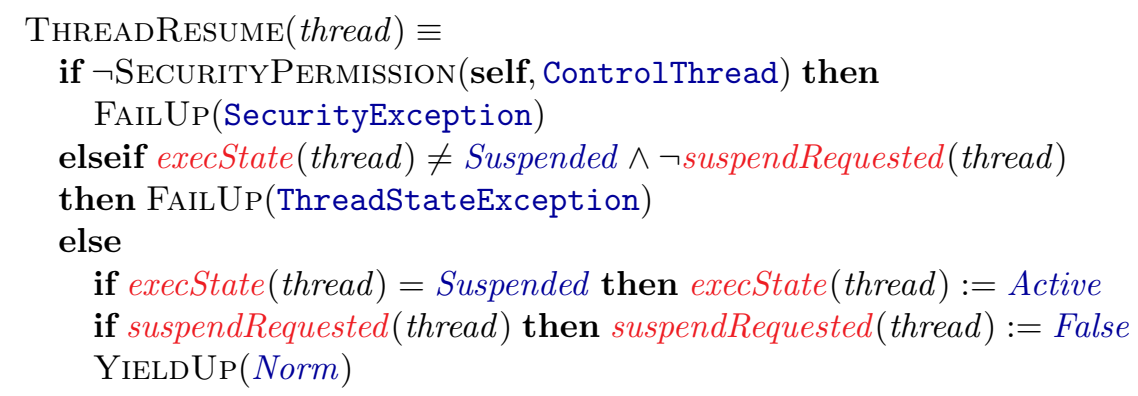

If a ThreadSuspend $(t)$ is executed in parallel with a ThreadResume $(t)$ on a thread $t$ that already has a suspend request, the resume has priority over the suspend. ${ }^{3}$

\footnotetext{
${ }^{3}$ One could as well forbid the parallel execution by a run constraint (see Sect. 4.1).
} 


\subsection{The methods of the Monitor class}

The methods of the Monitor class are static and are used to acquire and release locks of monitors. If they are invoked with the null reference, an exception is thrown. The Monitor.Enter method is used to acquire the lock of a monitor. If the current thread already owns the lock, the lockCount is increased. If the lock is free and the readyQueue of the monitor is empty, the thread immediately gets the lock. Otherwise, the thread changes its state from Active to Syncing and the thread is added to the readyQueue of the monitor. In case of a pending interrupt, a ThreadinterruptedException is thrown.

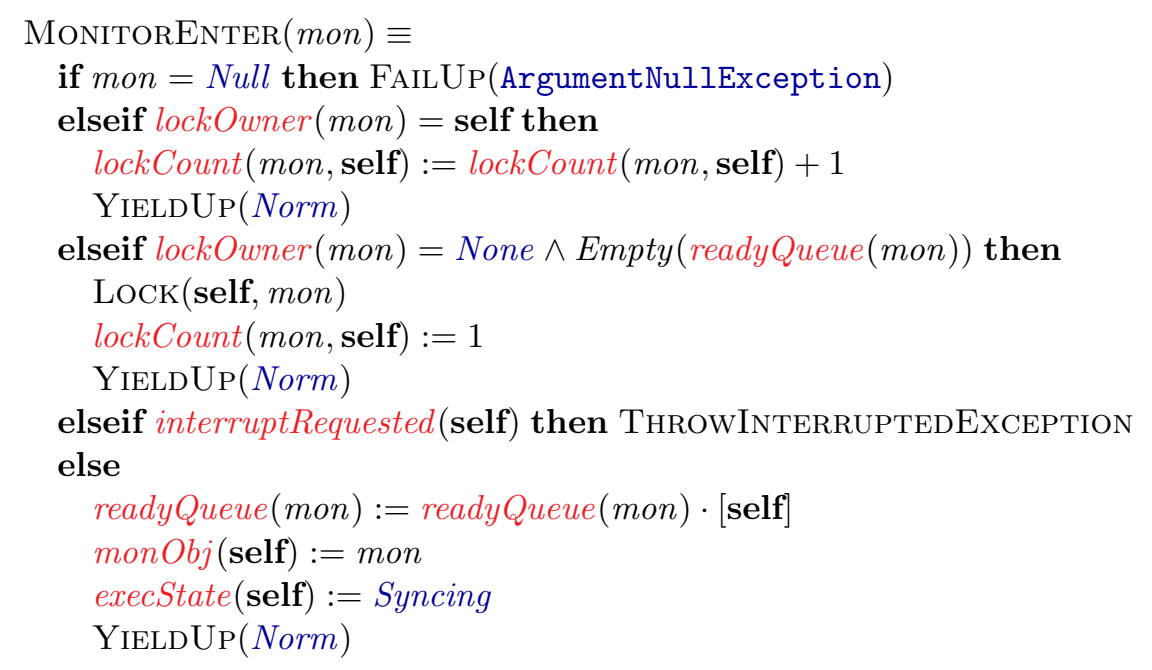

To lock a monitor means to update the lockOwner of the monitor.

$\operatorname{Lock}($ thread, mon $) \equiv$ lockOwner $($ mon $):=$ thread

The Monitor.Exit method decrements the lock count by one. If the lock count becomes 0 , the lock is released.

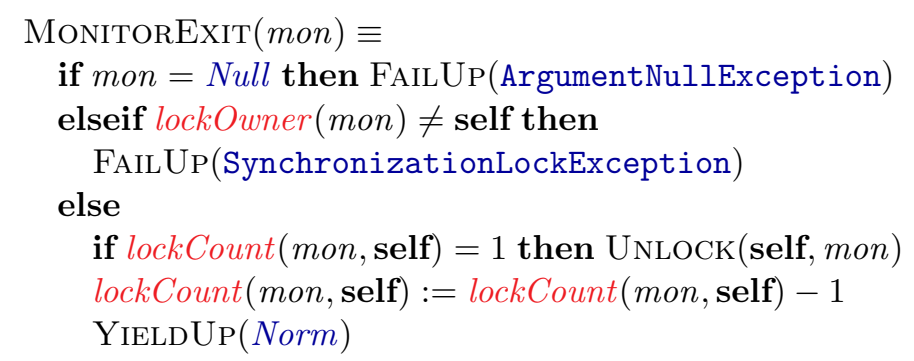

To release a lock means to update the lockOwner of the monitor to None.

$\operatorname{UNLOCK}($ thread, mon $) \equiv$ lockOwner $($ mon $):=$ None

The documentation [11] says that a thread can only exit a monitor if it owns the lock. The following code, however, runs in the .NET framework 1.1 as well as in Rotor [16] without throwing a SynchronizationLockException. 


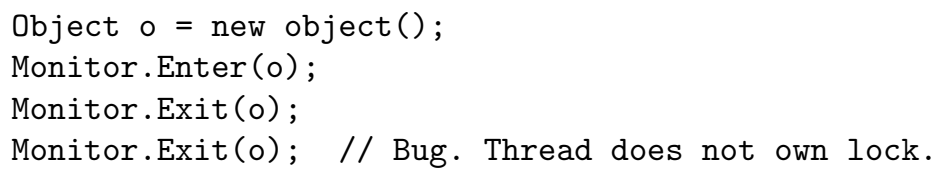

The Monitor.Wait method appends the current thread to the waitQueue of the monitor and temporarily releases the lock of the monitor. The execution state of the current thread is changed from Active to Waiting.

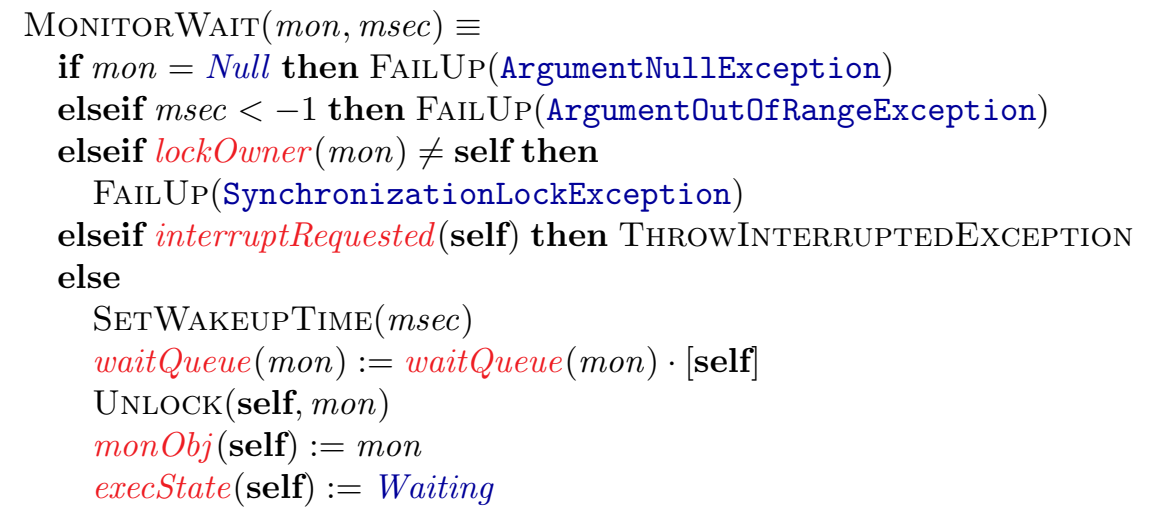

The thread remains in the waitQueue of the monitor until the monitor is pulsed or msec milliseconds have passed. At the return, the method indicates with a boolean result whether the time has expired.

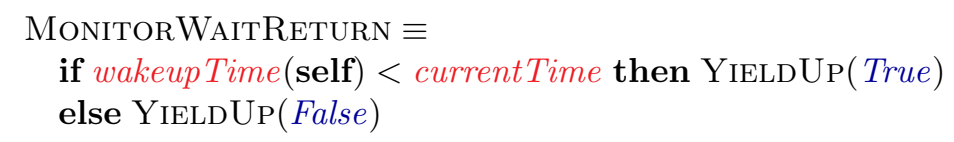

The Monitor.Pulse method moves the first element of the waitQueue of the monitor to the readyQueue. If the waitQueue is empty, the method just returns. Note, that we allow also that a thread with an abort or an interrupt request can be pulsed (this point is discussed also in [10]).

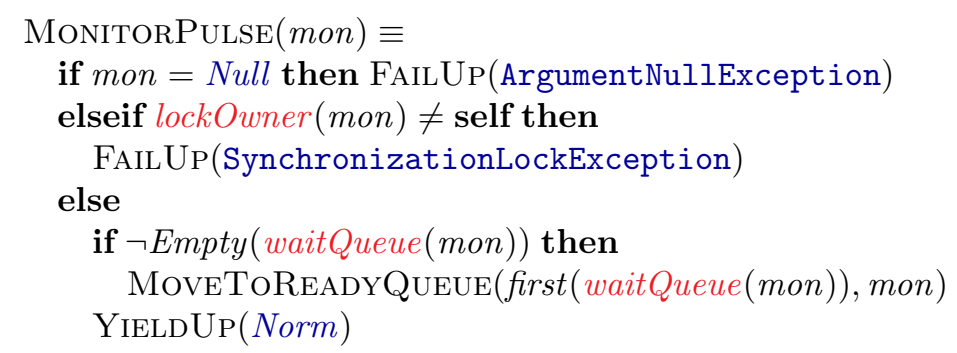

When a thread is moved from the waitQueue to the readyQueue, its execution state is changed from Waiting to Pulsed.

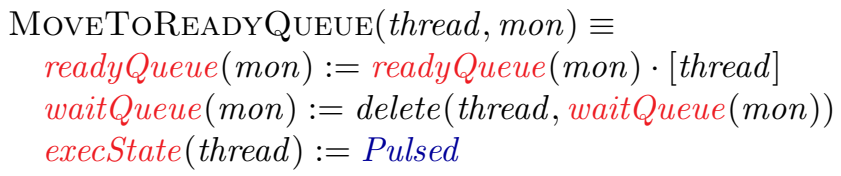


The Monitor. PulseAll methods moves all waiting threads into the readyQueue of the monitor.

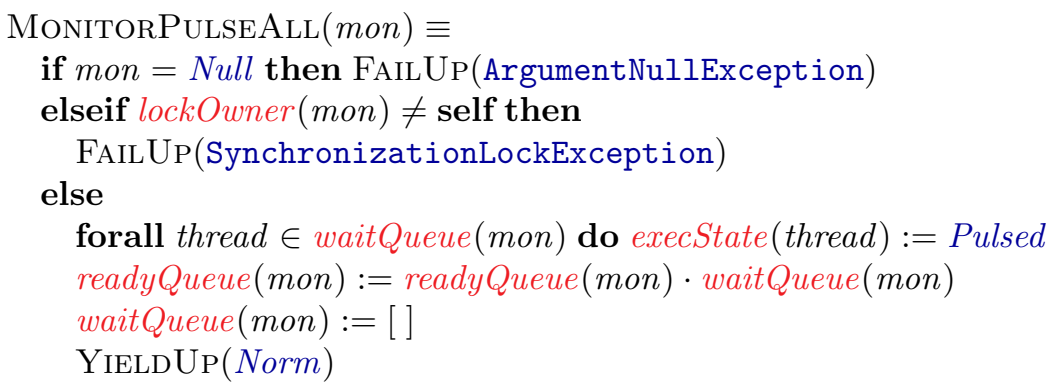

In Java, the wait and the ready queues are not FIFO queues but unordered sets. The Object.notify method of Java chooses an arbitrary element from the wait set of an object and it is not guaranteed that every thread in the wait set is ever chosen. The proposal for the new Java memory model [10] even allows so-called spurious wake-ups. This means that the system is allowed to remove a thread from the wait set of an object without any reason. Note, that the POSIX thread function pthread_cond_signal() is also allowed to wake up more than one thread.

\subsection{Scheduling of threads, timing, locking and asynchronous exceptions}

Although priorities can be assigned to threads in C\#, it is not guaranteed that they are honored by the scheduling algorithm. The main ASM rule for sequential $\mathrm{C} \#$ therefore chooses repeatedly one of the possible threads and executes one (small) step in the computation of the thread. In this way the computation steps of the currently running threads are interleaved.

EXECSEQUENTIALCSHARP $\equiv$ choose thread $\in$ Thread do EXECSTEP(thread)

The next computation step of a thread depends on its current execution state.

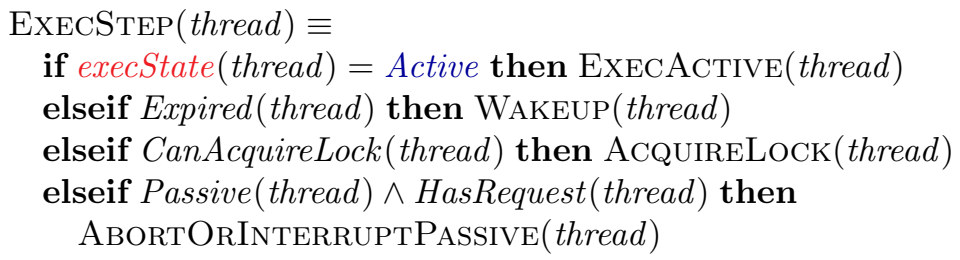

A thread is expired, if its wakeup time has been passed. The system time in milliseconds is given by the monitored function currentTime.

$$
\begin{aligned}
& \text { Expired }(\text { thread }) \Longleftrightarrow \text { execState }(\text { thread }) \in\{\text { Waiting, Sleeping, Joined }\} \wedge \\
& \text { wakeupTime }(\text { thread }) \leq \text { currentTime }
\end{aligned}
$$

A thread can acquire the lock, if it can acquire the lock of its current monitor object monObj that was set in MonitorEnter or MonitorWAit. 


$$
\text { CanAcquireLock(thread) } \Longleftrightarrow \text { CanAcquireLock(thread, monObj(thread)) }
$$

The lock of a monitor can be acquired, if the lock is free and the thread is the first thread in the readyQueue of the monitor.

$$
\begin{aligned}
& \text { CanAcquireLock }(\text { thread, mon }) \Longleftrightarrow \\
& \text { execState }(\text { thread }) \in\{\text { Syncing, Pulsed }\} \wedge \text { lockOwner }(\text { mon })=\text { None } \wedge \\
& \text { thread }=\text { first }(\text { readyQueue }(\text { mon }))
\end{aligned}
$$

A thread has a request, if it has an abort or an interrupt request.

$$
\text { HasRequest }(t) \Longleftrightarrow \text { abortRequested }(t) \vee \text { interruptRequested }(t)
$$

When the execution state of the chosen thread is Active, the next step in the computation of the thread is executed by the rule $\operatorname{ExECCsharp}($ thread). In case of a pending abort the system waits until the thread has left any finally block or catch clause before it aborts the threads. In case of a pending suspend, the system waits until the thread reaches a so-called safe point before suspending the thread. Safe points are points that are also safe for garbage collection.

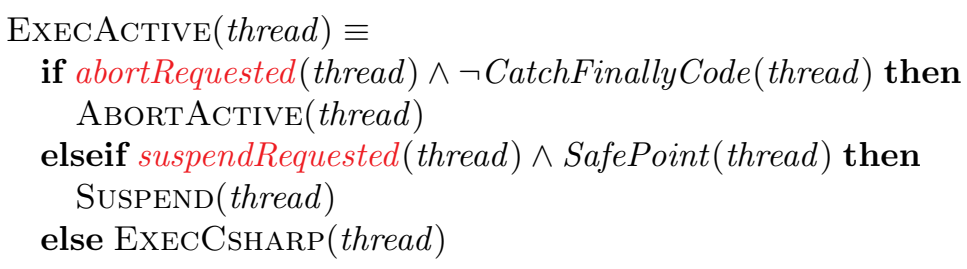

A thread aborts by throwing a ThreadAbortException. The fact that the thread is responding to the abort request is recorded in the abortInitiated flag.

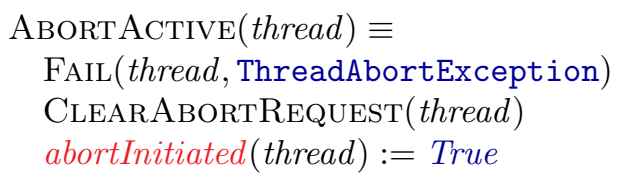

When the abort request is cleared, any pending interrupt request is also cleared.

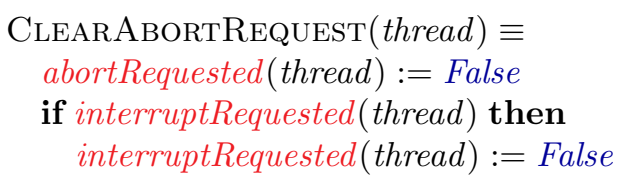

Like any other exception, a ThreadAbortException is propagated upwards in the frame stack of the thread. If it crosses a try block with catch clauses and a possible finally block, the catch clauses are searched for a matching handler. If there exists one, the corresponding catch block is executed. The finally block is executed afterwards. At the end of the catch block, however, the ThreadAbortException is re-thrown by the system. More precisely, if an abort has been initiated and a catch block terminates but not with an exception, then a new ThreadAbortException is thrown at the end of the catch block. If the catch block terminates abruptly with an exception, that exception is propagated upwards. Hence, the rules for exception handling of $\mathrm{C} \# \mathcal{E}$ of [2] have to be refined. If the current position of the thread (indicated by the black triangle) is at the end of a catch block with result res and res is not an exception, a 
ThreadAbortException is thrown. Hence a ThreadAbortException cannot be swallowed unless the Thread. ResetAbort method is called (see Sect. 3.3).

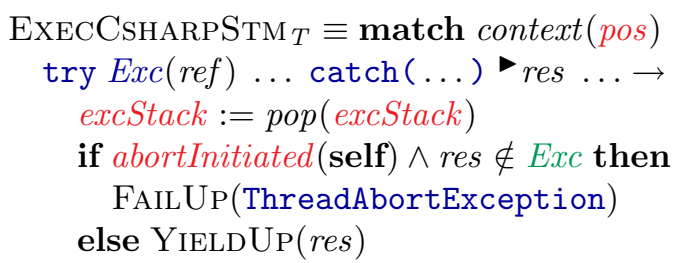

When a suspend request is pending and the thread has reached a safe point, the system changes its execution state from Active to Suspended and clears the request.

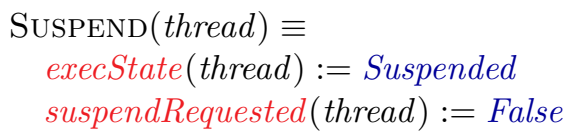

If a sleeping, joined or waiting thread has expired, the system has to wakeup the thread. If the thread is Sleeping, its execution state is changed to Active. If the thread is Joined, it is removed from the joinSet and returns from the Thread.Join method (see Sect. 3.3). If the thread is Waiting it is moved to the readyQueue of the monitor and has to re-acquire the lock (its state is changed to Pulsed).

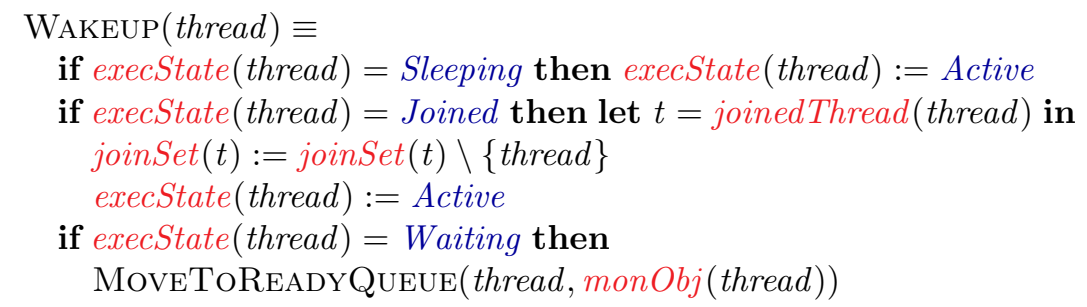

If a thread can acquire the lock, it becomes the owner of the lock. Its execution state is changed from Pulsed or Syncing to Active. If it acquires the lock for the first time, the lockCount is initialized to 1. Otherwise, when the thread has temporarily released the lock by invoking the Monitor.Wait method, the old lock count is still valid.

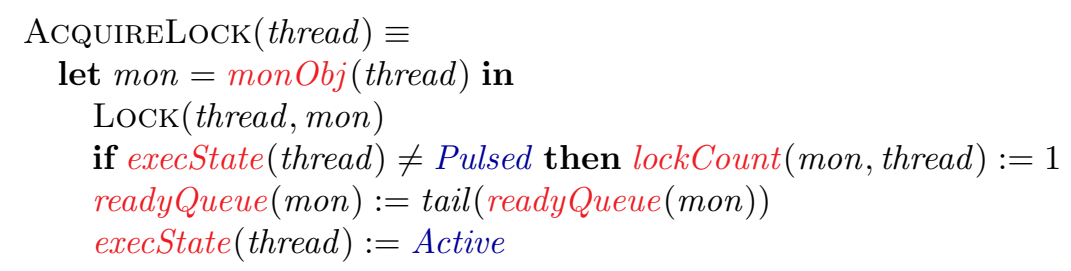

If a passive thread has an abort or an interrupt request, an exception is injected into the thread. If both, an abort request and an interrupt request are pending, the abort request has priority (unless the thread executes catch or finally code).

$$
\begin{aligned}
& \text { ABORTORINTERRUPTPASSIVE }(\text { thread }) \equiv \\
& \text { if abortRequested }(\text { thread }) \wedge \neg \text { CatchFinallyCode }(\text { thread }) \text { then }
\end{aligned}
$$




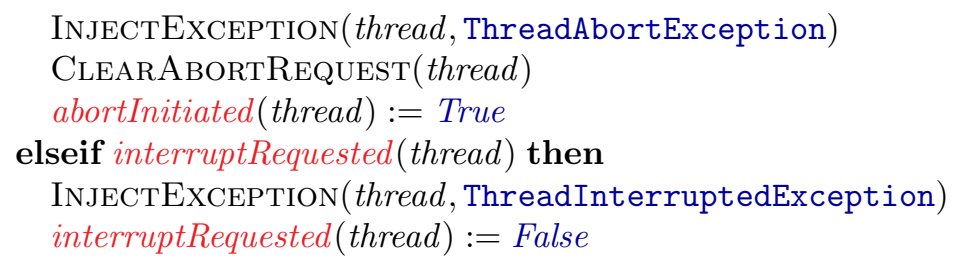

Injecting a ThreadAbortException or a ThreadInterruptedException into a thread means to create a new excpetion object and to force the thread to throw the exception (using FAIL). If the thread is Syncing, Sleeping or Joined, its execution state is changed to Active in order that the exception can propagate upwards and probably terminate the thread. If the thread is Waiting, it is moved to the readyQueue and has to re-acquire the lock, since in this case the thread is still in a critical section of code and possible exception handlers and finally blocks should only be executed under the exclusive control of the monitor. If the thead is Pulsed, its execution state is not updated, since the thread has to re-acquire the lock before propagating the exception.

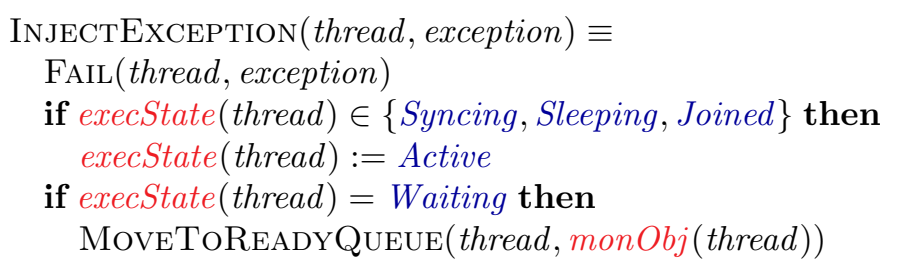

A thread terminates when the frame stack of the thread is empty again and the Invoke method of the delegate of the thread terminates. The method can terminate normally or abruptly with an exception. In any case, the execution state of the thread is updated from Active to Dead and the threads that are joined are notified by changing their states from Joined to Active such that they can return from the Thread. Join method. If the thread terminates with an exception, the exception may or may not be reported as unhandled exception to the console.

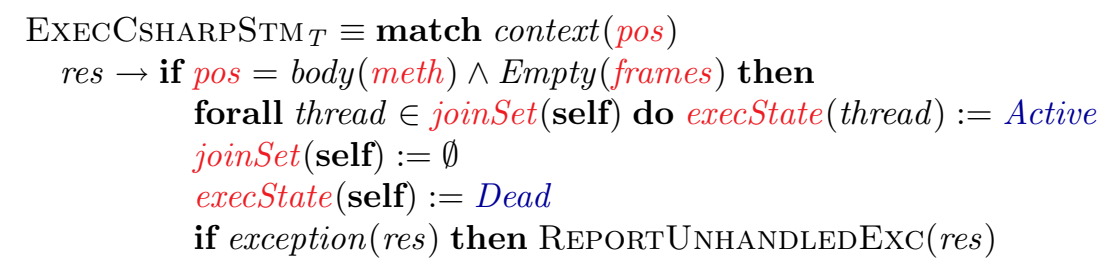

When a thread is Dead, it remains in this execution state and cannot be reactivated (see also Fig. 4).

\section{A parallel model for $\mathrm{C} \#$ threads}

On a multiprocessor system different threads can execute code concurrently on different processors. We model this case using a special kind of distributed ASMs that are executable in tools like AsmL [6]. The main rule for the parallel thread 
model chooses repeatedly an arbitrary set of possible threads and executes in parallel the next computation step for each of the chosen threads.

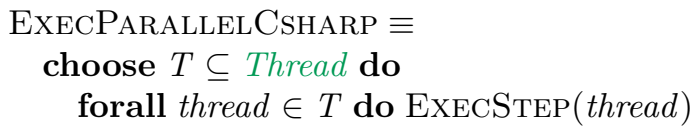

The ExecStep rule is the same as in the sequential model. However, since the computation steps are executed in parallel, conflicts can occur if the same location is updated by different threads to different values. Updates of the local state of a thread (frame stack, program counter, local environment, operand stack) are not critical, since the local state is parameterized by the thread (see Sect. 3.1). Updates to the shared memory are discussed in Sect. 5 below. An analysis of the transition rules shows that the following conflicts have to be avoided by imposing run constraints on EXECPARALLELCSHARP.

\subsection{Run constraints for the parallel thread model}

1. The rule AcQuireLock(thread) is not allowed to run in parallel with the rule MonitorEnter $($ mon $)$, if monObj $($ thread $)=$ mon. Otherwise, there would be a conflict for lockOwner (mon).

2. It is not allowed that two different threads execute $\operatorname{MonitorEnTER}(m o n)$ in parallel. Otherwise, conflicts for lockOwner(mon) and readyQueue(mon) could occur.

3. The rules AcquireLock(thread) and MoveToReadyQueue $(t$, mon) are not allowed to run in parallel, if monObj $($ thread $)=$ mon. Otherwise, there is a conflict for readyQueue(mon).

4. The rule MonitorPulse(mon) or MonitorPulseAll(mon) is not allowed to run in parallel with the rule MoveToReadyQueue $(t, m o n)$. Otherwise, there would be a conflict for readyQueue (mon).

5. MoveToReadyQueue $\left(t_{1}\right.$, mon $)$ and MoveToReadyQueue $\left(t_{2}, m o n\right)$ are not allowed to run in parallel for $t_{1} \neq t_{2}$. Otherwise, there would be a conflict for readyQueue(mon).

6. The rules Suspend (thread) and ThreadResume(thread) are not allowed to run in parallel. Otherwise, the ThreadResume(thread) would be ignored by the system.

Note, that MoveToReadyQueue $(t, m o n)$ is used in the rules $\operatorname{Wakeup}(t)$ and AbortOrInterruptPassive $(t)$ in case that the execution state of $t$ is Waiting.

\section{$5 \quad$ The .NET memory model}

The .NET memory model is outlined in [5, Partition $1, \S 11.6 .5, \S 11.6 .7]$. According to $[13,15]$, it gives the following (weak) guarantees about the ordering of memory reads and writes:

- Reads and writes from the same thread to a location cannot be re-ordered.

- No read can move before a lock acquire (or volatile read). 
- No write can move after a lock release (or volatile write).

- Writes cannot cross a Thread.WriteMemoryBarrier().

- Neither reads nor writes can cross a Thread.MemoryBarrier().

To application programmers the memory model is often (wrongly) explained in a stronger form. Each thread has its local cache. After acquiring the lock the thread's cache is invalidated, so that reads afterward are done from the main memory. After releasing the lock the thread's cache is flushed to main memory. Note that in .NET a read or write of a volatile location affects also the ordering of reads and writes of other locations.

For the ASM specification of the .NET memory model we follow the ASM specification of the Local Consistency Memory Model for Java in [1] and use a universe of events that is divided into disjoint subuniverses as follows:

\section{Event $::=$ WriteEvent $\mid$ LockEvent $\mid$ UnlockEvent | ReadVolatileEvent | BarrierEvent| WriteBarrierEvent}

Events are ordered during a run of a multi-threaded C\# program by a dynamic predicate $\prec$. We denote by $\prec^{+}$the transitive closure and by $\prec^{*}$ the reflexive, transitive closure of $\prec$. Each WriteEvent has two attributes, an address and a value, adr: WriteEvent $\rightarrow$ Address val: WriteEvent $\rightarrow$ Value. The latest event of a thread is recorded in latest: Thread $\rightarrow$ Event.

The memory model is now reduced to the question: "Which write event(s) can be seen by a memory read?" When a thread writes a value to an address, a new WriteEvent is created.

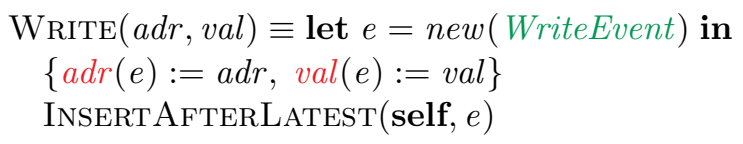

The new WriteEvent is inserted in the event order immediately after the latest event of the current thread.

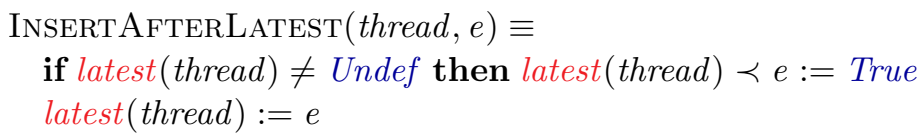

Reading a value from an address means choosing an appropriate WriteEvent for that address and returning the value that has been written to that address.

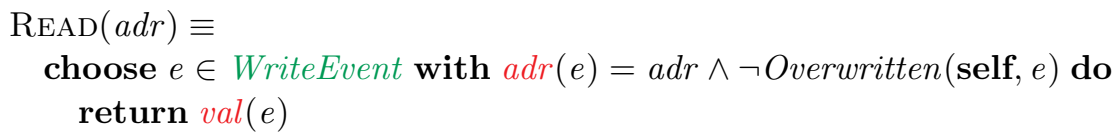

A read cannot see arbitrary write events but only those that are not overwritten with respect to the latest event of the current thread or any memory barrier.

$$
\begin{aligned}
& \text { Overwritten }(t, e) \Longleftrightarrow \\
& \quad \exists w \in \operatorname{WriteEvent}\left(\operatorname{adr}(w)=\operatorname{adr}(e) \wedge e \prec^{+} w \wedge \operatorname{Previous}(t, w)\right) \\
& \text { Previous }(t, w) \Longleftrightarrow w \prec^{*} \text { latest }(t) \vee \exists b \in \operatorname{BarrierEvent}\left(w \prec^{*} b\right)
\end{aligned}
$$




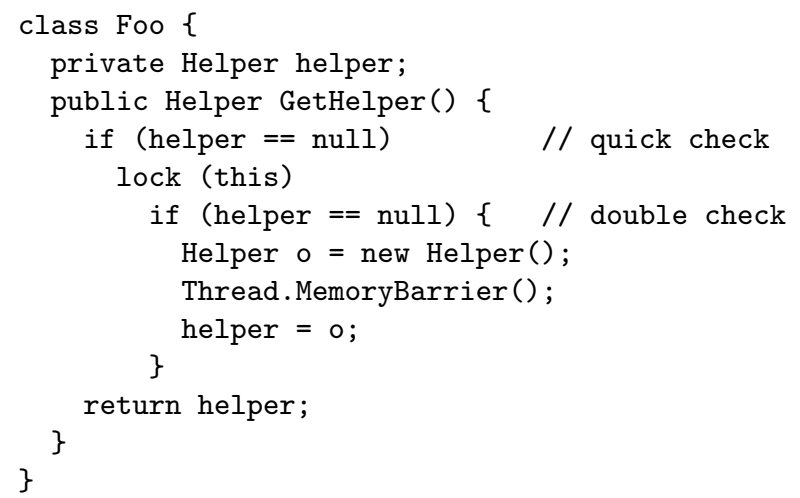

Fig. 6. The double checked locking pattern.

When a monitor is locked a new LockEvent is created and inserted in the event order after the latest UnlockEvent of the monitor as well as after the latest event of the current thread (in this way the write events of the last thread that owned the lock are synchronized with the current thread).

$$
\begin{aligned}
& \text { LOCK }(\text { thread, mon }) \equiv \text { let } e=\text { new }(\text { LockEvent }) \text { in } \\
& \text { if latestUnlock }(\text { mon }) \neq \text { Undef then latestUnlock }(\text { mon }) \prec e:=\text { True } \\
& \text { InSERTAFTERLATEST }(\text { thread, } e) \\
& \text { forall } b \in \text { WriteBarrierEvent } \mathbf{d o} b \prec e:=\text { True } \\
& \text { lockOwner }(\text { mon }):=\text { thread }
\end{aligned}
$$

The function latestUnlock: Monitor $\rightarrow$ UnlockEvent records the latest unlock event of a monitor. The UnlockEvent created at the monitor exit prevents overwritten write events from being seen by the next thread that aquires the lock of the monitor.

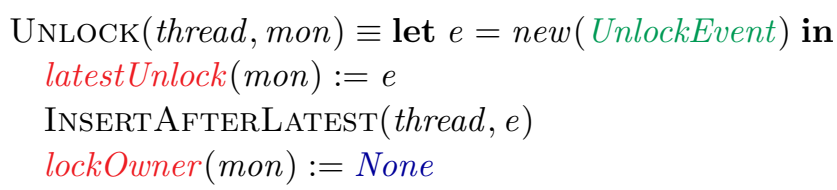

The function Thread.MemoryBarrier creates a new BarrierEvent which is inserted in the event order after the latest event of the current thread. (Barrier events are used in the definition of the Overwritten predicate above.)

$$
\begin{aligned}
& \text { MemoryBARRIER } \equiv \text { let } e=\text { new (BarrierEvent) in } \\
& \text { InSERTAFTERLATEST }(\text { self }, e)
\end{aligned}
$$

The function Thread.WriteMemoryBarrier creates a WriteBarrierEvent which are inserted in the event order before any future lock event.

$$
\begin{aligned}
& \text { WrITEMEMORYBARRIER } \equiv \text { let } e=n e w(\text { WriteBarrierEvent }) \text { in } \\
& \text { INSERTAFTERLATEST }(\text { self }, e)
\end{aligned}
$$


A read of a volatile field creates a new ReadVolatileEvent. The choosen write event (which was a volatile write) is inserted in the event ordering before the read event.

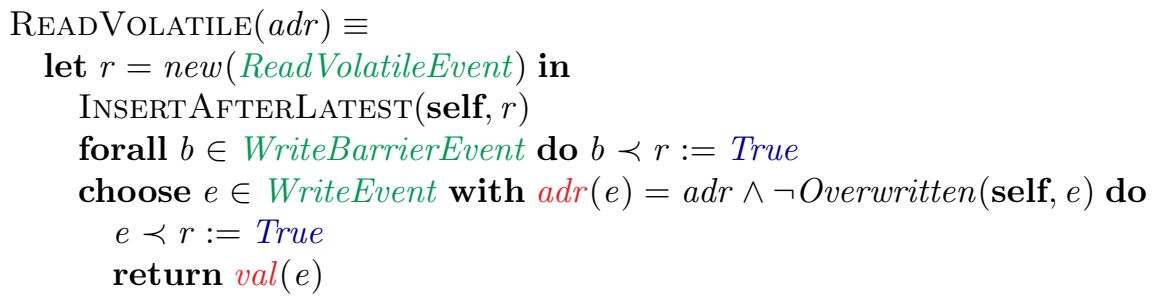

A write to a volatile field uses the normal WRITE rule.

The so-called double-checked locking pattern in Fig. 6 uses a memory barrier to prevent another thread from seeing a non-null value of the helper field while the fields of the Helper object itself still contain their default null values which are overwritten in the constructor of the Helper class. (The constructor may be inlined by the JIT compiler.) Instead of using the memory barrier the helper field could be declared volatile.

It is not clear to us, whether the following example is allowed by the Ecma .NET memory model. Consider two threads that concurrently execute the following instructions, where initially $p \cdot x==0, p \cdot y==0$ :

Thread $1 \quad$ Thread 2

$\mathrm{r} 1=\mathrm{p} \cdot \mathrm{x} ; \quad \mathrm{r} 2=\mathrm{p} \cdot \mathrm{y} ;$

$\mathrm{p} \cdot \mathrm{y}=1 ; \quad \mathrm{p} \cdot \mathrm{x}=2$;

Is the result $r 1==2$ and $r 2==1$ possible? According to our specification of the memory model, it is not possible. However, if we allow the compiler to switch the assignments in both threads (under the assumption that p.x and p.y are independent variables), the result is plausible. Maybe the result is justified by the paragraph about execution order in $[4, \S 10.10]$.

\section{Conclusion}

The ASM method forces the person who writes a specification to think in terms of an abstract implementation. This leads to questions and cases that are usually forgotten in other formal or informal approaches. Fig. 7 contains a bug in Microsoft's .NET Framework 1.1 [11] which was detected during the construction of our thread model. The bug shows a situation where a thread executes code in a critical sections proteced by a monitor without owning the lock of the monitor.

The main function in Fig. 7 creates an account, starts another thread with the Deposit method of the account and sleeps for 100 milliseconds. During the sleep, the deposit thread acquires the lock of the account and waits on the account in order to later deposit 100 dollars when it is pulsed. After the sleep, the main thread locks the account and executes its critical section. At the beginning, the balance is still 0 . The main thread pulses the account and moves 


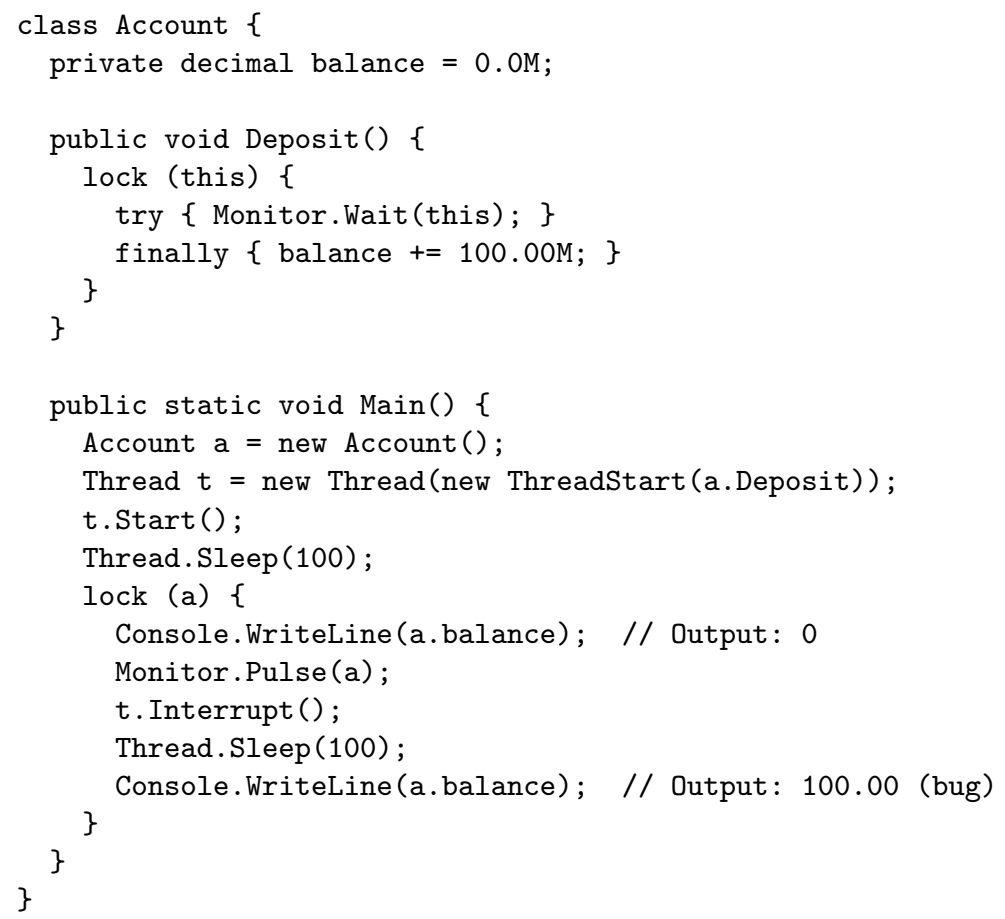

Fig. 7. A bug in Microsoft's .NET Framework version 1.1

the deposit thread from the wait queue into the ready queue of the account. Then it interrupts the deposit thread and sleeps again for 100 milliseconds (still holding the lock of the account). When it awakes, the balance has changed to 100. OM. Why?

The change of the balance is only possible if the deposit thread executes the finally block, which is in its critical section, without owning the lock of the account. The same problem occurs if Thread. Interrupt is replaced by Thread.Abort. The Rotor SSCLI implementation [16] correctly prints 0 at the end of the lock statement in the main function. After that, however, it deadlocks for unknown reasons.

\section{Acknowledgement}

We gratefully acknowledge partial support of this work by a Microsoft grant within the ROTOR project during the year 2002-2003.

\section{References}

1. V. Awhad and C. Wallace. A unified formal specification and analysis of the new Java memory models. In E. Börger, A. Gargantini, and E. Riccobene, editors, 
Abstract State Machines 2003-Advances in Theory and Applications, volume 2589 of Lecture Notes in Computer Science, pages 166-185. Springer-Verlag, 2003.

2. E. Börger, G. Fruja, V. Gervasi, and R. F. Stärk. A high-level modular definition of the semantics of C\#. Theoretical Computer Science, 2004. To appear.

3. E. Börger and R. F. Stärk. Abstract State Machines-A Method for High-Level System Design and Analysis. Springer-Verlag, 2003.

4. C\# Language Specification. Standard ECMA-334, 2001. Web pages at http: //www.ecma-international.org/.

5. Common Language Infrastructure (CLI). Standard ECMA-335, 2001. Web pages at http://www . ecma-international.org/.

6. Foundations of Software Engineering Group, Microsoft Research. AsmL. Web pages at http://research.microsoft.com/foundations/AsmL/, 2001.

7. J. Gosling, B. Joy, G. Steele, and G. Bracha. The Java(tm) Language Specification. Addison Wesley, second edition, 2000.

8. Y. Gurevich. Evolving algebras 1993: Lipari guide. In E. Börger, editor, Specification and Validation Methods, pages 9-36. Oxford University Press, 1993.

9. A. Hejlsberg, S. Wiltamuth, and P. Golde. C\# Language Specification. AddisonWesley, 2003.

10. Java(TM) Memory Model and Thread Specification Revision. Web pages at http: //www.jcp.org/en/jsr/detail?id=133.

11. Microsoft .NET Framework 1.1 Software Development Kit. Download from http: //msdn.microsoft.com/netframework/howtoget/.

12. The Mono project. Web pages at http://www.go-mono.com/.

13. V. Morrison. The DOTNET Memory Model. dotnet@discuss.develop.com mailing list, 2002.

14. J. Richter. Safe thread synchronization. MSDN Magazine, .NET column, January 2003.

15. A. G. Robison. Memory consistency \& .NET. Dr. Dobb's Journal, April 2003.

16. Rotor - Shared Source Common Language Infrastructure (SSCLI). Web pages at http://msdn.microsoft.com/net/sscli/ and http://www.sscli.net/.

17. R. F. Stärk, J. Schmid, and E. Börger. Java and the Java Virtual MachineDefinition, Verification, Validation. Springer-Verlag, 2001.

18. D. Stutz, T. Neward, and G. Shilling. Shared Source CLI Essentials. O'Reilly, 2003.

19. C. von Praun and T. Gross. Static conflict analysis for multi-threaded objectoriented programs. In PLDI 2003, pages 115-128, June 2003. 
The following invariants are satisfied (where $t \in$ Thread and mon $\in$ Ref):

(thread) $x \in$ Thread $\Longleftrightarrow$ execState $(x) \neq$ Undef

(join1) execState $(t) \in\{$ Unstarted, Dead $\} \Longrightarrow$ joinSet $(t)=\emptyset$

(join2) execState $(t)=$ Joined $\Longrightarrow t \in$ joinSet $($ joinedThread $(t))$

(join3) $x \in$ joinSet $(t) \Longrightarrow x \in$ Thread $\wedge$ execState $(x)=$ Joined

(wait1) execState $(t)=$ Waiting $\Longrightarrow$

$t \in$ waitQueue $(\operatorname{monObj}(t)) \wedge \operatorname{lock} \operatorname{Count}(\operatorname{monObj}(t), t) \geq 1$

(wait2) $x \in$ waitQueue $($ mon $) \Longrightarrow x \in$ Thread $\wedge \operatorname{exc}$ State $(x)=$ Waiting

(sync) execState $(t)=$ Syncing $\Longrightarrow$

$t \in \operatorname{ready} Q u e u e(\operatorname{monObj}(t)) \wedge \operatorname{lockCount}(\operatorname{monObj}(t), t) \in\{\operatorname{Undef}, 0\}$

(pulse) execState $(t)=$ Pulsed $\Longrightarrow$

$t \in \operatorname{readyQueue}(\operatorname{monObj}(t)) \wedge \operatorname{lockCount}(\operatorname{monObj}(t), t) \geq 1$

(ready) $x \in \operatorname{readyQueue}($ mon $) \Longrightarrow$

$x \in$ Thread $\wedge$ execState $(x) \in\{$ Syncing, Pulsed $\}$

(lock1) lockCount (mon, $x) \neq$ Undef $\Longrightarrow x \in$ Thread

(lock2) execState $(t)=$ Unstarted $\Longrightarrow \operatorname{lockCount}($ mon, $t)=$ Undef

(lock3) lockOwner $($ mon $) \neq$ None $\Longrightarrow$

lockOwner $($ mon $) \in$ Thread $\wedge \operatorname{lockCount~}($ mon, $\operatorname{lockOwner}($ mon $)) \geq 1$

(lock4) lockCount $($ mon, $t) \geq 1 \wedge \operatorname{execState}(t)=$ Active $\Longrightarrow$ lockOwner $($ mon $)=t$

(abort) abortInitiated $(t) \Longrightarrow \neg$ abortRequested $(t)$

(suspend) execState $(t)=$ Suspend $\Longrightarrow$

$\neg$ suspendRequested $(t) \wedge \neg$ abortRequested $(t)$

(time) execState $(t) \in\{$ Waiting, Sleeping, Joined $\} \Longrightarrow$ wakeupTime $(t) \geq 0$

The most important invariant is (lock4) which says that among the threads that have entered a given monitor only the thread that owns the lock of the monitor can be active at any time. Hence, the locks are exclusive and can be used to protect critical sections. 